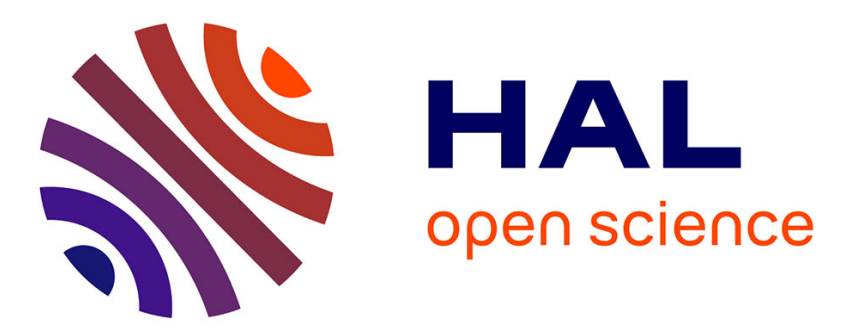

\title{
Robust supervised classification with mixture models: Learning from data with uncertain labels
}

\author{
Charles Bouveyron, Stéphane Girard
}

\section{To cite this version:}

Charles Bouveyron, Stéphane Girard. Robust supervised classification with mixture models: Learning from data with uncertain labels. Pattern Recognition, 2009, 42 (11), pp.2649-2658. 10.1016/j.patcog.2009.03.027 . hal-00325263v2

\section{HAL Id: hal-00325263 \\ https://hal.science/hal-00325263v2}

Submitted on 12 Feb 2009

HAL is a multi-disciplinary open access archive for the deposit and dissemination of scientific research documents, whether they are published or not. The documents may come from teaching and research institutions in France or abroad, or from public or private research centers.
L'archive ouverte pluridisciplinaire $\mathbf{H A L}$, est destinée au dépôt et à la diffusion de documents scientifiques de niveau recherche, publiés ou non, émanant des établissements d'enseignement et de recherche français ou étrangers, des laboratoires publics ou privés. 


\title{
Robust supervised classification with mixture models: Learning from data with uncertain labels
}

\author{
Charles Bouveyron ${ }^{1} \&$ Stéphane GirarD ${ }^{2}$ \\ ${ }^{1}$ SAMOS-MATISSE, CES, UMR CNRS 8174, \\ University Paris 1 (Panthéon-Sorbonne) \\ ${ }^{2}$ MISTIS, INRIA Rhône-Alpes \& LJK
}

\begin{abstract}
In the supervised classification framework, human supervision is required for labeling a set of learning data which are then used for building the classifier. However, in many applications, human supervision is either imprecise, difficult or expensive. In this paper, the problem of learning a supervised multiclass classifier from data with uncertain labels is considered and a modelbased classification method is proposed to solve it. The idea of the proposed method is to confront an unsupervised modelling of the data with the supervised information carried by the labels of the learning data in order to detect inconsistencies. The method is able afterward to build a robust classifier taking into account the detected inconsistencies into the labels. Experiments on artificial and real data are provided to highlight the main features of the proposed method as well as an application to object recognition under weak supervision.
\end{abstract}

Key words: supervised classification, data with uncertain labels, mixture models, robustness, label noise, weakly-supervised classification.

\section{Introduction}

In the supervised classification framework, human supervision is required to associate labels with a set of learning observations in order to construct a classifier. However, in many applications, this kind of supervision is either imprecise, difficult or expensive. For instance, in bio-medical applications, domain experts are asked to manually label a sample of learning data (MRI images, DNA micro-array, ...) which are then used for building a supervised classifier. The cost of the supervision phase is usually high due to the 
difficulty of labeling complex data. Furthermore, an human error is always possible in such a difficult task and an error in the supervision phase could have big effects on the decision phase, particularly if the size of the learning sample is small. It is therefore very important to provide supervised classifiers robust enough to deal with data with uncertain labels.

\subsection{The label noise problem}

In statistical learning, it is very common to assume that the data are noised. Two types of noise can be considered in supervised learning: the noise on the explanatory variables and the noise on the response variable. Noise on explanatory variables has been widely studied in the literature whereas the problem of noise on the response variable has received less attention in some supervised situations. While almost all approaches model the noise on the response variable in regression analysis (see Chap. 3 of [15] for details), label noise remains an important and unsolved problem in supervised classification. Brodley and Friedl summarized in [7] the main reasons for which label noise can occur. Since the main assumption of supervised classification is that the labels of learning samples are true, existing methods giving a full confidence to the labels of the learning data naturally provide disappointing classification results when the learning dataset contains some wrong labels. Particularly, model-based discriminant analysis methods such as Linear Discriminant Analysis (LDA, see Chap. 3 of [21]) or Mixture Discriminant Analysis (MDA, see [14]) are sensitive to label noise. This sensitivity is mainly due to the fact that these methods estimate the model parameters from the learning data and label noise naturally perturbs these estimates.

\subsection{Related works}

Learning a supervised classifier from data with uncertain labels can be achieved using three main strategies: cleaning the data, using robust estimations of model parameters and finally modelling the label noise.

Data cleaning approaches. Early approaches tried to clean the data by removing the misclassified instances using some kind of nearest neighbor algorithm $[10,12,30]$. Other works treat the noisy data using the C4.5 algorithm [17, 32], neural networks [31] or a saturation filter [11]. Hawkins et al. [16] identified as outliers the data subset whose deletion leads to the smallest value of the determinant of the within-group covariance matrix. Guyon 
et al. proposed in [13] to remove noisy observations with a cumulative information criterion and further checking by human experts. However, removing noisy instances could decrease the classification bias but also increase the classification variance since the cleaned dataset is of smaller size than the original one. Thus, this procedure could give a less efficient classifier than the classifier built with noisy data when the number of learning data is small.

Robust estimation of model parameters. Therefore, other researchers proposed not to remove any learning instance and to build instead supervised classifiers robust to label noise. Bashir et al. [2] focused on robust estimation of the model parameters in the mixture model context. Maximum likelihood estimators of the mixture model parameters are replaced by the corresponding S-estimators (see Rousseeuw and Leroy [25] for a general account on robust estimation) but the authors only observed a slight reduction of the average probability of misclassification. Similarly, Mingers [23], Sakakibara [26] and Vannoorenberghe et al. [29] proposed noise-tolerant approaches to make decision tree classifiers robust to label noise. Boosting [24, 27] can also be used to limit the sensitivity of the built classifier to the label noise.

Noise modelling. Among all these solutions, the model proposed in [18] by Lawrence et al. has the advantage of explicitely including the label noise in the model with a sound theoretical foundation in the binary classification case. Denoting by $y$ and $\tilde{y}$ the actual and the observed class labels of an observation $x$, it is assumed that their joint distribution can be factorised as $p(x, y, \tilde{y})=P(y \mid \tilde{y}) p(x \mid y) P(\tilde{y})$. The class conditional densities $p(x \mid y)$ are modelled by Gaussian distributions while the probabilistic relationship $P(y \mid \tilde{y})$ between noisy and observed class labels is specified by a $2 \times 2$ probability table. An EM-like algorithm is introduced for building a kernel Fisher discriminant classifier on the basis of the above model. This work was recently extended by $\mathrm{Li}$ et al. in [19] who proposed a new incorporation of the noise model in the classifier and relaxed the distribution assumption of Lawrence et al. by allowing each class density $p(x \mid y)$ to be modeled by a mixture of several Gaussians.

\subsection{The proposed approach}

We propose in this paper a supervised classification method, called Robust Mixture Discriminant Analysis (RMDA), designed for dealing with label noised data. Conversely to the noise modelling methods, the approach 
proposed in this paper does not rely on a specific label noise model which could be ill adapted in some situations. The main idea of our approach is to compare the supervised information given by the learning data with an unsupervised modelling based on the Gaussian mixture model. With such an approach, if some learning data have wrong labels, the comparison of the supervised information with an unsupervised modelling of the data allows to detect the inconsistent labels. It is possible afterward to build a supervised classifier by giving a low confidence to the learning observations with inconsistent labels. The main advantages of the proposed approach compared to previous works are the explicit modelling of more than two classes and the flexibility of the method due to the use of a global mixture model.

The remainder of this paper is organized as follows. The model of the proposed method is presented in Section 2 and Section 3 is devoted to the inference aspects. Experimental studies on simulated and real datasets are reported in Section 4. Finally, an application to object recognition under weak supervision is presented in Section 5.

\section{Robust mixture discriminant analysis}

In order to compare the supervised information given by the learning data with an unsupervised modelling, we propose to use an unsupervised mixture model in which the supervised information is introduced.

\subsection{The mixture model}

Let us consider a mixture model in which two different structures coexist: an unsupervised structure of $K$ clusters (represented by the random discrete variable $S$ ) and a supervised structure, provided by the learning data, of $k$ classes (represented by the random discrete variable $C$ ). As in the standard mixture model, we assume that the data $\left(x_{1}, \ldots, x_{n}\right)$ are independent realizations of a random vector $X \in \mathbb{R}^{p}$ with density function:

$$
p(x)=\sum_{j=1}^{K} P(S=j) p(x \mid S=j),
$$

where $P(S=j)$ is the prior probability of the $j$ th cluster and $p(x \mid S=j)$ is the corresponding conditional density. Let us now introduce the supervised 
information carried by the learning data. Since $\sum_{i=1}^{k} P(C=i \mid S=j)=1$ for all $j=1, \ldots, K$, we can plug this quantity in (1) to obtain:

$$
p(x)=\sum_{i=1}^{k} \sum_{j=1}^{K} P(C=i \mid S=j) P(S=j) p(x \mid S=j),
$$

where $P(C=i \mid S=j)$ can be interpreted as the probability that the $j$ th cluster belongs to the $i$ th class and thus measures the consistency between classes and clusters. Using the classical notations of parametric mixture models and introducing the notation $r_{i j}=P(C=i \mid S=j)$, we can reformulate (2) as follows:

$$
p(x)=\sum_{i=1}^{k} \sum_{j=1}^{K} r_{i j} \pi_{j} p(x \mid S=j),
$$

where $\pi_{j}=P(S=j)$. Therefore, (3) exhibits both the "modelling" part of our approach, based on the mixture model, and the "supervision" part through the parameters $r_{i j}$. Since the modelling introduced in this section is based on the mixture model, we can use any conditional density to model each cluster. In particular, the use of the Gaussian mixture model is discussed below.

\subsection{The case of Gaussian mixture models}

Among all parametric densities, the Gaussian model is probably the most used in classification. The Gaussian mixture model has been studied extensively in the last decades and used in many situations (see [1] for a review).

Usual Gaussian model. In the case of the usual Gaussian mixture model, the conditional density $p(x \mid S=j)$ is modelled by a Gaussian density $\phi$ with mean $\mu_{j}$ and covariance $\Sigma_{j}$. Under this assumption, (3) can therefore be rewritten as:

$$
p(x)=\sum_{i=1}^{k} \sum_{j=1}^{K} r_{i j} \pi_{j} \phi\left(x ; \mu_{j}, \Sigma_{j}\right) .
$$

Parsimonious Gaussian models. In some situations, modelling the data with a full covariance matrix can be too expensive in terms of number of parameters to estimate. In such a case, it is possible to make additional assumptions on the structure of the covariance matrix. For example, in the well-known Linear Discriminant Analysis (LDA) method, the covariance matrices of the 
different components are supposed to be equal to a unique covariance matrix (common Gaussian model hereafter). It is also possible to assume that the covariance matrix of each mixture component is diagonal (diagonal Gaussian model) or proportional to the identity matrix (spherical Gaussian model). These models are known as parsimonious Gaussian models in the literature since they require to estimate less parameters than the classical Gaussian model. Celeux and Govaert proposed in [8] a family of parsimonious Gaussian models based on an eigenvalue decomposition of the covariance matrix including the previous models. These parsimonious Gaussian models were then applied in [4] to supervised classification.

Gaussian models for high-dimensional data. Nowadays, many scientific domains produce high-dimensional data like medical research (DNA microarrays) or image analysis (see Section 5 for an illustration). Classifying such data is a challenging problem since the performance of classifiers suffers from the curse of dimensionality [3]. Classification methods based on Gaussian mixture models are directly penalized by the fact that the number of parameters to estimate grows up with the square of the dimension. It is then necessary to use parsimonious Gaussian models in order to obtain stable classifier. However, these parsimonious models are usually too constrained to correctly fit the data in a high-dimensional space. To overcome this problem, Bouveyron et al. proposed recently in [5] a family of Gaussian models adapted to high-dimensional data. This approach, based on the idea that high-dimensional data live in low-dimensional spaces, assumes that the covariance matrix of each mixture component has only $d_{j}+1$ different eigenvalues where $d_{j}$ is the dimension of the subspace of the $j$ th mixture component. This specific modelling allows to deal with the situation where the class manifold and the data density could be not correlated in high-dimensional spaces.

\subsection{Link with Mixture Discriminant Analysis}

It is possible to establish a link between model (4) and the supervised method Mixture Discriminant Analysis (MDA) [14] in which each class is modelled by a mixture of $K_{i}$ Gaussian densities. Denoting by $K=\sum_{i=1}^{k} K_{i}$ the total number of Gaussian components and keeping in mind the notations of Paragraph 2.1, MDA assumes that the conditional density of the $i$ th class, 
$i=1, \ldots, k$, is:

$$
p(x \mid C=i)=\sum_{j=1}^{K} \pi_{i j} \phi\left(x ; \mu_{j}, \Sigma_{j}\right),
$$

where $\pi_{i j}=P(C=i, S=j)$ is the prior probability of the $j$ th mixture component of the $i$ th class. Note that $\pi_{i j}=0$ if the $j$ th mixture component is not included in the $i$ th class. Moreover, remarking that $\pi_{i j}=r_{i j} \pi_{j}$, we obtain

$$
p(x)=\sum_{i=1}^{k} \sum_{j=1}^{K} r_{i j} \pi_{j} \phi\left(x ; \mu_{j}, \Sigma_{j}\right),
$$

which formally corresponds to model (4). The main difference is that, in the MDA case, the labels are certain (supervised context). Thus, $r_{i j}=P(C=$ $i \mid S=j)$ is known and reduces to $r_{i j}=1$ if the $j$ th mixture component belongs to the $i$ th class and $r_{i j}=0$ otherwise. Consequently, in the case where the labels of learning data are all consistent with the modelling of these data, RMDA should provide the same classifier as MDA.

\subsection{Classification step}

In model-based discriminant analysis, new observations are usually assigned to a class using the maximum a posteriori (MAP) rule. The MAP rule assigns a new observation $x$ to the class for which $x$ has the highest posterior probability. Therefore, the classification step mainly consists in calculating the posterior probability $P(C=i \mid X=x)$ for each class $i=1, \ldots, k$. In the case of the model described in this section, this posterior probability can be expressed as follows using the Bayes' rule:

$$
P(C=i \mid X=x)=\sum_{j=1}^{K} r_{i j} P(S=j) p(x \mid S=j) / p(x),
$$

and, since $P(S=j \mid X=x)=P(S=j) p(x \mid S=j) / p(x)$, we finally obtain:

$$
P(C=i \mid X=x)=\sum_{j=1}^{K} r_{i j} P(S=j \mid X=x) .
$$

Therefore, the classification step of RMDA relies on (7) and requires the estimation of the probabilities $r_{i j}$ as well as the unsupervised classification probabilities $P(S=j \mid X=x)$. As we can see, the probabilities $r_{i j}$, which 
quantify the consistency between the groups and the classes, balance the importance of the groups in the final classification rule. Consequently, the classifier associated with this decision rule will be mainly based on the groups which are very likely to be made of points from a unique class.

\section{Estimation procedure}

Due to the nature of the model proposed in Section 2, the estimation procedure is made of two steps corresponding respectively to the unsupervised and to the supervised part of the comparison. The first step consists in estimating the parameters of the mixture model in an unsupervised way leading to the clustering probabilities $P(S=j \mid X=x)$. In the second step, the parameters $r_{i j}$ linking the mixture model with the information carried by the labels of the learning data are estimated by maximization of the likelihood.

\subsection{Estimation of the mixture parameters}

In this first step of the estimation procedure, the labels of the data are discarded to form $K$ homogeneous groups. Therefore, this step consists in estimating the parameters of the chosen mixture model.

Usual and parsimonious Gaussian models. In the case of the usual Gaussian model, the classical procedure for estimating the proportions $\pi_{j}$, the means $\mu_{j}$ and the variance matrices $\Sigma_{j}$, for $j=1, \ldots, K$, is the Maximum Likelihood (ML) method. Unfortunately, it is not possible to find directly a solution of the maximum likelihood problem. In such a case, the ExpectationMaximization (EM) algorithm proposed by Dempster et al. (1977) provides the ML estimates of the parameters using an iterative procedure. We refer to [4] for the parameter estimation in the case of parsimonious Gaussian models.

Gaussian models for high-dimensional data. If the chosen mixture model involves Gaussian models for high-dimensional data, this step consists in estimating the following model parameters: the proportions $\pi_{j}$, the means $\mu_{j}$, the subspace variances $\left(a_{1 j}, \ldots, a_{d_{j} j}\right)$, the noise variance $b_{j}$, the subspace orientation matrix $Q_{j}$ and the subspace dimension $d_{j}$ for each mixture component. In [5], an EM-like approach is presented to estimate the parameters $\pi_{j}, \mu_{j}, a_{i j}, b_{j}$ and $Q_{j}$. An empirical strategy based on the eigenvalue scree 
is also proposed in this work to find the intrinsic dimension of each mixture component. We refer to [5] for more details and to Section 5 for an application to object regognition.

\subsection{Estimation of the parameters $r_{i j}$}

In this second step of the procedure, the labels of the data are introduced to estimate the $k \times K$ matrix of parameters $R=\left(r_{i j}\right)$ and we use the parameters learned in the previous step as the mixture parameters. The parameters $r_{i j}$ modify the unsupervised model for taking account of the label information. They thus indicate the consistency of the unsupervised modelling of the data with the supervised information carried by the labels of the learning data. Since we consider a supervised problem, the labels $c_{1}, \ldots, c_{n}$ of the learning data $x_{1}, \ldots, x_{n}$ are known, and we can therefore introduce $\mathcal{C}_{i}=\left\{x_{\ell}, \ell=1, \ldots, n / c_{\ell}=i\right\}$. From (7), the log-likelihood associated to our model can be expressed as:

$$
\begin{aligned}
\ell(R) & =\sum_{i=1}^{k} \sum_{x \in \mathcal{C}_{i}} \log P(X=x, C=i), \\
& =\sum_{i=1}^{k} \sum_{x \in \mathcal{C}_{i}} \log \left(\sum_{j=1}^{K} r_{i j} P(S=j \mid X=x)\right)+\xi,
\end{aligned}
$$

where, in view of $(1), \xi=\sum_{i=1}^{k} \sum_{x \in \mathcal{C}_{i}} \log p(x)$ does not depend on $R$. This relation is matricially rewritten as:

$$
\ell(R)=\sum_{i=1}^{k} \sum_{x \in \mathcal{C}_{i}} \log \left(R_{i} \Psi(x)\right)+\xi,
$$

with the $\mathbb{R}^{K}$-vector $\Psi(x)=(P(S=1 \mid X=x), \ldots, P(S=K \mid X=x))^{t}$ and where $R_{i}$ is the $i$ th row of $R$. Consequently, we end up with a constrained optimization problem:

$$
\begin{cases}\operatorname{maximize} & \sum_{i=1}^{k} \sum_{x \in \mathcal{C}_{i}} \log \left(R_{i} \Psi(x)\right), \\ \text { with respect to } & r_{i j} \in[0,1], \forall i=1, \ldots, k, \forall j=1, \ldots, K, \\ \text { and } & \sum_{i=1}^{k} r_{i j}=1, \forall j=1, \ldots, K\end{cases}
$$

Since it is not possible to find an explicit solution to this optimization problem, an iterative optimization procedure has to be used to compute the maximum likelihood estimators of the parameters $r_{i j}$. The partial derivatives 
associated with (8) can be expressed as follows:

$$
\frac{\partial \ell(R)}{\partial r_{i j}}=\sum_{x \in \mathcal{C}_{i}} \frac{\Psi_{j}(x)}{R_{i} \Psi(x)},
$$

where $\Psi_{j}(x)$ is the $j$ th coordinate of $\Psi(x)$. This optimum search has been implemented in Matlab using the function fmincon which is designed to find a constrained optimum of multivariate functions.

\subsection{Model selection and complexity}

We now focus on the problem of choosing the most appropriate mixture model for RMDA. In the context of this work, this issue includes the selection of the conditional density of each mixture component as well as the choice of the number of sub-classes per mixture component. We discuss biefly as well the complexity and the scalability of RMDA.

Choosing a mixture model. As discussed in Paragraph 2.2, it is sometimes useful to use a parsimonious model or a model designed for high-dimensional data depending on the nature of the data and the size of the training dataset. In order to choose among all existing models, it is possible to use either cross-validation or information criteria, such as the Bayesian Information Criterion (BIC) [28]. However, the practician has to be careful when choosing between both approaches. Indeed, choosing cross-validation implies that more importance is given to the labels of the learning dataset and this can be contrary to the idea that there is label noise in the data. On the other hand, adopting an information criterion, such as BIC, implies that more importance is given to the unsupervised modelling of the data.

Choosing the number of sub-classes. Selecting the number of sub-classes is usually a complex problem since different classes can have a different number of mixture components. For instance, in the case of MDA, trying out all combinations of component numbers will confront the practician to a computational problem with a high level of complexity. In [14], the authors proposed to make the additional assumption that the numbers of mixture components of the classes are equal but this could be far away from the truth. Due to the nature of the RMDA model introduced in this paper, choosing the number of sub-classes per mixture component reduces to choosing the total number of clusters $K$. Indeed, the selection of the number of sub-classes per class 
will be implicitely done through the parameters $r_{i j}$ which measures the consistency between the sub-classes and the mixture components. Therefore, it only remains to select the unique parameter $K$ and this easier problem can be also addressed using cross-validation or BIC (with similar implications).

Complexity and scalability of RMDA. Regarding the model complexity, RMDA mainly depends on the chosen mixture model since RMDA requires only to estimate the mixture parameters and the parameters $r_{i j}$. The number of parameters $r_{i j}$ to estimate is $(k-1) K$ and thus does not depend on the dimension of the data. Conversely, the number of mixture parameters to estimate heavily depends on the data dimension. For instance, the number of parameters to estimate in a Gaussian mixture of $K$ classes in $\mathbb{R}^{p}$ is larger than $K p^{2} / 2$. Therefore, when the data dimension becomes high, it is preferable to switch for a parsimonious model which requires the estimation of less parameters. In particular, Gaussian models for high-dimensional data (see Paragraph 2.2) require only the estimation of approximatively $K p d / 2$ parameters, where $d$ is the intrinsic data dimension. Therefore, these models are well suited for classifying high-dimensional data as soon as $d$ is small compared to the original data dimension $p$. Regarding now the scalability, RMDA inherits its flexibility and its ability to model complex processes from MDA. This feature is mainly due to the modelling of each class by a mixture of several Gaussians in order to be able to deal with non-Gaussian data.

\section{Experimental results}

In this section, we present experimental results on artificial and real datasets in situations illustrating the problem of supervised classification under uncertainty.

\subsection{Experimental setup}

In the following studies, we consider the general problem of label switching between the classes. In this case, complex models are very sensitive but parsimonious models can also be affected if the contamination rate is high. In order to simulate a label noise, the observation labels have been switched following a Bernoulli distribution with parameter $\eta$ ranging from 0 to 1 and representing the contamination rate. Each label $\ell$ is therefore left unchanged with probability $1-\eta$ or switches to a value $\ell^{\prime} \neq \ell$ with probability $\eta / k$. In all studies, the performance of the methods is assessed by the correct 


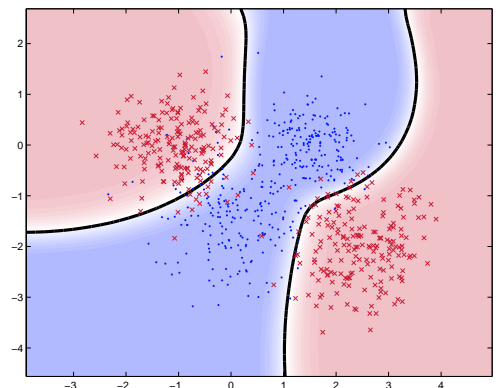

MDA with $\eta=0$

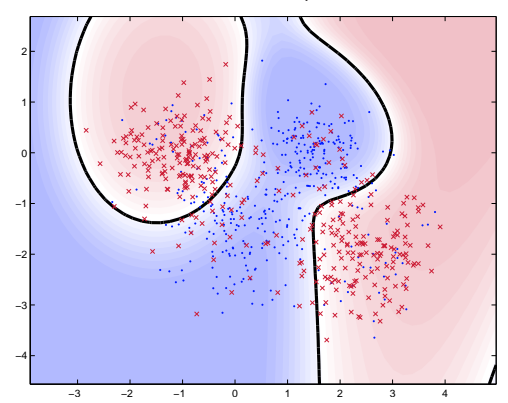

MDA with $\eta=0.15$

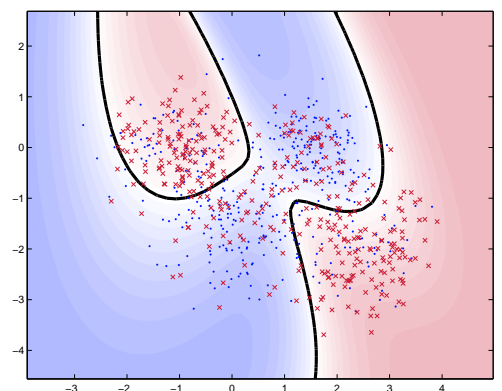

MDA with $\eta=0.30$

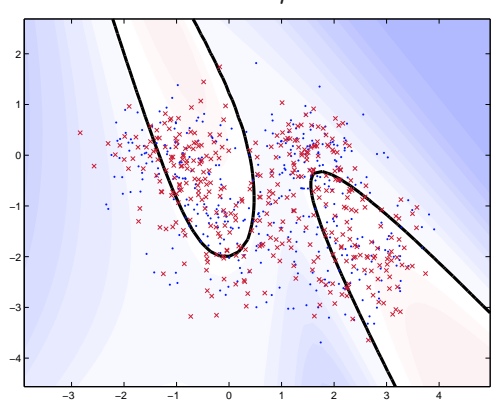

MDA with $\eta=0.45$

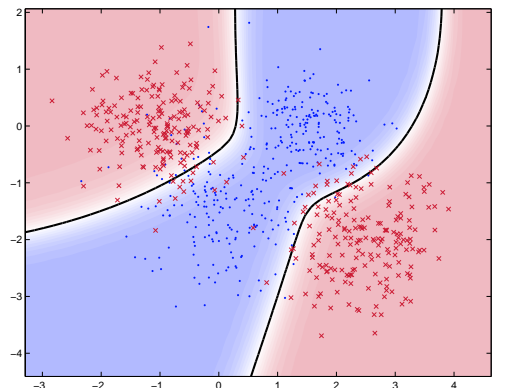

RMDA with $\eta=0$

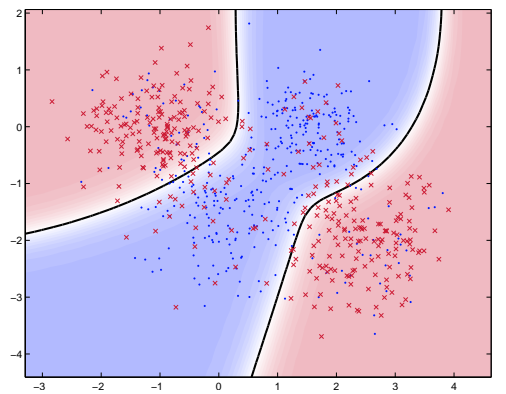

RMDA with $\eta=0.15$

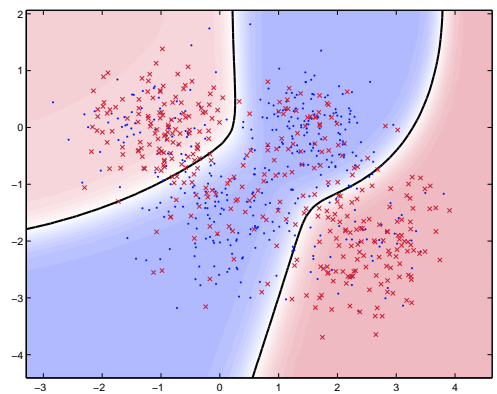

RMDA with $\eta=0.30$

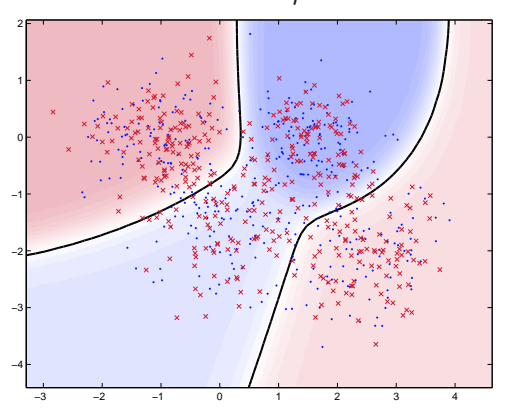

RMDA with $\eta=0.45$

Figure 1: Decision rules for MDA and RMDA for increasing contamination rates $\eta$ on a 2-dimensional simulated dataset (2 classes). 


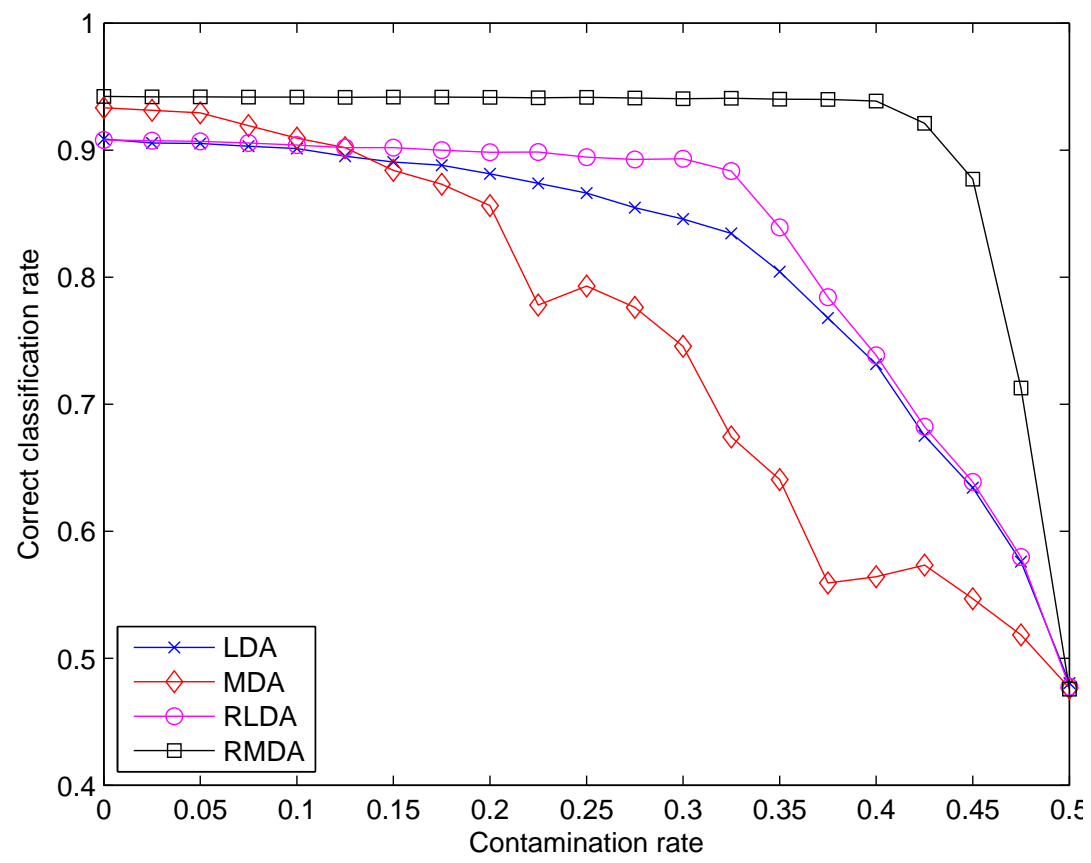

Figure 2: Performance of LDA, MDA, RLDA and RMDA for increasing contamination rates on a simulated dataset (2 classes).

classification rate and computed on a test dataset. The experiments have been repeated 25 times in order to average the classification results.

\subsection{Binary classification (simulated data)}

For this first experiment, we simulated the data following the mixture model of MDA and RMDA. The dataset is made of 2 classes and each class was modeled with a Gaussian mixture of 2 components. We used for the mixture components of each class a spherical Gaussian model. The means of the different mixture components were chosen in order to obtain two separated enough classes. Figure 1 compares the performance of MDA and RMDA (introduced in this paper) on a 2-dimensional simulated dataset for increasing contamination rates $\eta$. As expected, MDA and RMDA give similar decision rules when there is no label noise $(\eta=0)$. This observation confirms the existing link between both methods (see Paragraph 2.3). For higher contamination rates $\eta$, MDA builds very unstable decision rules whereas RMDA demonstrates its robustness by providing very stable decision boundaries. 


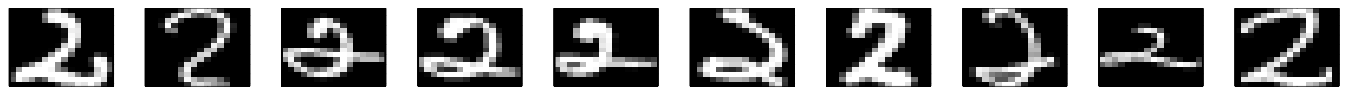 4941446444}

Figure 3: Some examples of the USPS-24 dataset.

The performance of LDA, MDA, RLDA (proposed by Lawrence et al. (2001)) and RMDA on a 25-dimensional dataset simulated under similar conditions is illustrated on Figure 2. First, it appears that RMDA is as efficient as MDA when there is no label noise and this illustrates again the equivalence between both methods in this special case. On the one hand, LDA and MDA appear to be sensitive to contamination. Particularly, MDA becomes very unstable for contamination rates higher than 0.2 . The behavior of these two supervised methods is not surprising since they both have full confidence in the labels of the data. On the other hand, RLDA turns out to be more robust than LDA but its performance quickly decreases for contamination rates higher than 0.3 . Finally, RMDA appears to be particularly robust for a large panel of contamination rates (up to 0.4 ).

\subsection{Binary classification (real data)}

We consider here a dataset from the real world, called USPS-24, extracted from the well-known USPS dataset ${ }^{1}$. The learning dataset is made of 1383 hand-written digits. Among them, 731 observations belong to the class of the digit 2 and of 652 observations belong to the class of the digit 4. Similarly, the test dataset contains 298 elements: 198 and 200 observations respectively from the classes of the digit 2 and 4 . These two classes have been chosen since they have a high misclassification rate in the original USPS dataset. Each observation of the USPS-24 dataset corresponds to a $16 \times 16$ grey level image of a digit and represented as a 256-dimensional vector. Figure 3 shows some samples from the dataset. For both MDA and RMDA, each class was modeled by a mixture of 5 Gaussians and, due to the high dimension of the data, we used for each mixture component a spherical Gaussian model. Figure 4 shows the performance of LDA, MDA, RLDA and RMDA on the USPS-24 dataset for increasing contamination rates. As in the previous experiment,

\footnotetext{
${ }^{1}$ The USPS dataset is available for download at www.kernel-machines.org.
} 


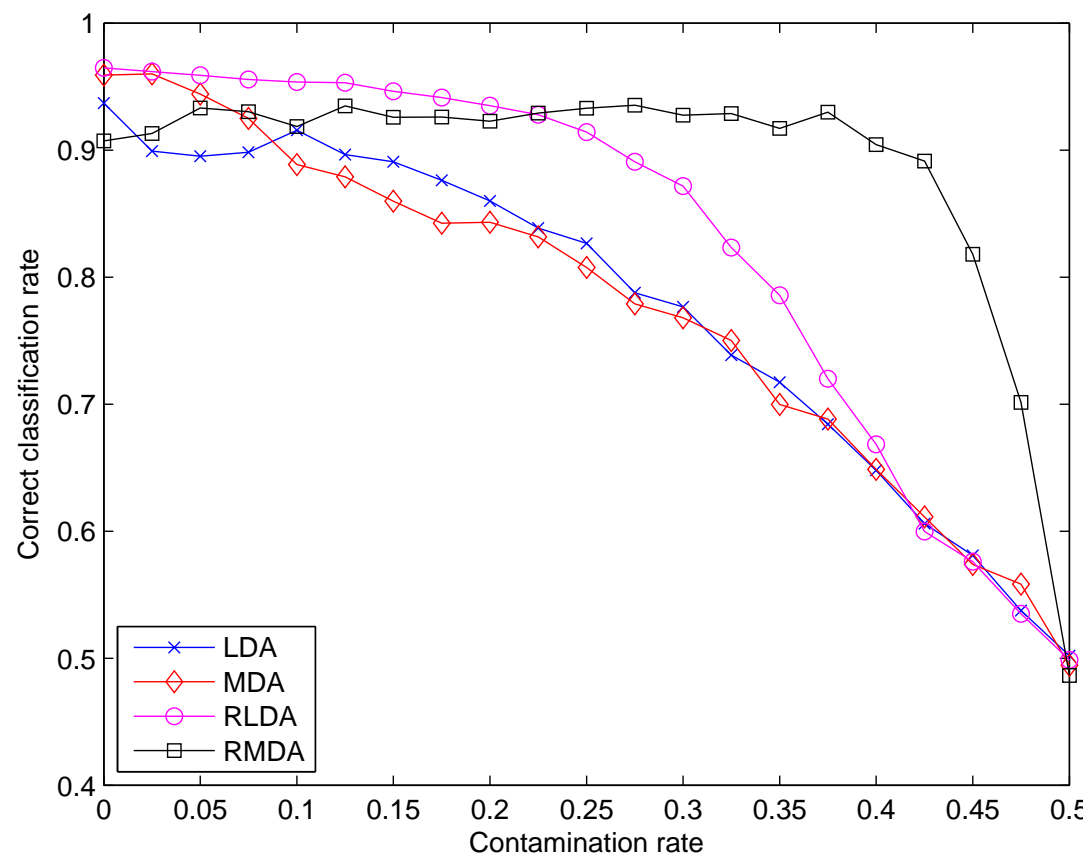

Figure 4: Performance of LDA, MDA, RLDA and RMDA for increasing contamination rates on the USPS-24 dataset.

LDA and MDA appear to be very sensitive to contamination. RLDA is again more robust than LDA and MDA but its performance decreases quickly for contamination rates higher than 0.2. Finally, RMDA appears to be very robust for contamination rates up to 0.4 and to be almost as efficient as the other methods when the label noise is low. In this experiment, RMDA has therefore demonstrated its ability to deal with label noise in real and complex situations.

\subsection{Multi-class classification (simulated data)}

This last simulation study aims at demonstrating the ability of RMDA to deal with label noise in multi-class classification problem whereas the existing methods consider only binary classification. Indeed, the model of RLDA, proposed by Lawrence et al. [18] and its extension [19] are designed for only two classes. We therefore compare here RMDA to only LDA and MDA which are able to deal with multi-class classification problems as well. As before, we simulated the data following the mixture model of MDA and RMDA. 


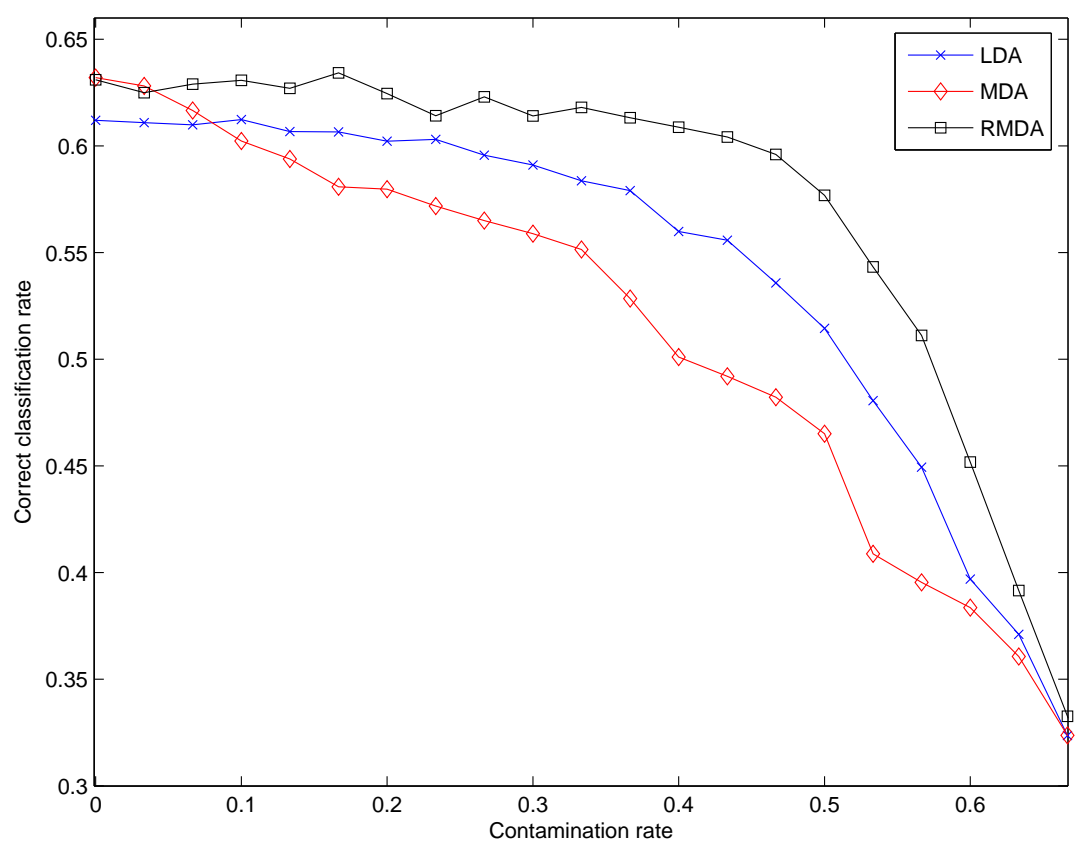

Figure 5: Performance of LDA, MDA and RMDA for increasing contamination rates on a simulated dataset (3 classes).

The simulated dataset is made of 3 classes and each class was modeled with a Gaussian mixture of 2 components in a 25-dimensional space. We used for the mixture components of each class a spherical Gaussian model. The means of the different mixture components were again chosen in order to obtain separated enough classes. Figure 5 shows the performance of LDA, MDA and RMDA for increasing contamination rates. Unsurprisingly, it can be noticed that the average correct classification rate of the methods is lower than in the binary classification case and decreases to $1 / 3$. Secondly, it appears that RMDA is again as efficient as MDA when there is no label noise. Regarding the robustness of the studied methods, LDA and MDA have similar behaviours as in the binary case. The performance of MDA decreases almost linearly with the contamination rate. As in the previous experiments, LDA is more robust than MDA for low contamination rates but its performance decreases after 0.2. Finally, RMDA turns out to be as robust as in the binary case since its performance is almost constant for contamination rates up to 0.45 . This experiment illustrates the robustness 
of RMDA in the multi-class classification case which is a more common and difficult problem in practice than the binary classification problem.

\section{Application to object recognition under weak supervision}

The supervised classification method proposed in this work is designed for performing classification in the presence of label noise. We present here an application of this method to object recognition under weak supervision.

\subsection{Object recognition under weak supervision}

Object recognition is one of the most challenging problems in computer vision and it requires that human experts segment a very large number of images for each object category. Earlier approaches characterized the objects by their global appearance and were not robust to occlusion, clutter and geometric transformations. To avoid these problems, recent methods use local image descriptors for selecting the relevant parts of the images and then classify these local descriptors to one of the object categories. Regarding the supervision, it is clear that it is impossible that human experts segment images for each object category given the infinite number of existing object categories. However, it is easy to obtain images containing a given object (using Google Image for instance) and to assume that all descriptors of these images are representative of the studied object even though we know that it is wrong. By doing that, we consciously introduce a label noise between the class "object" and the class "background" but, using the approach proposed in this paper, it should be possible to identify all pixels which actually belong to the class "object" and, finally, localize the studied object in the images. This approach will be called in the sequel weakly-supervised object recognition.

\subsection{The data}

The object category database used in this study is the Pascal dataset [9] which has been proposed for an object localization challenge organized by the Pascal Network. Examples of images in this dataset are presented in Figure 6. The Pascal dataset is divided into four categories: motorbikes, bicycles, people and cars. It contains 684 images for learning and two test sets: test 1 (689 images) and test 2 (956 images). Images in test 1 were collected from the same distribution as the training images. The set test 2 can be seen as a harder challenge since images are collected by "Google Image" and thus 


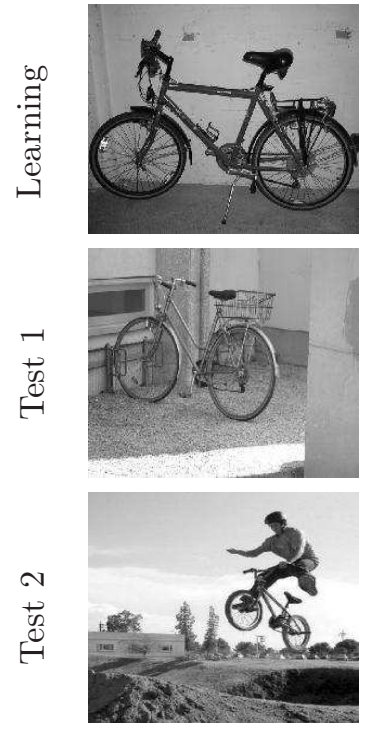

Bicycle
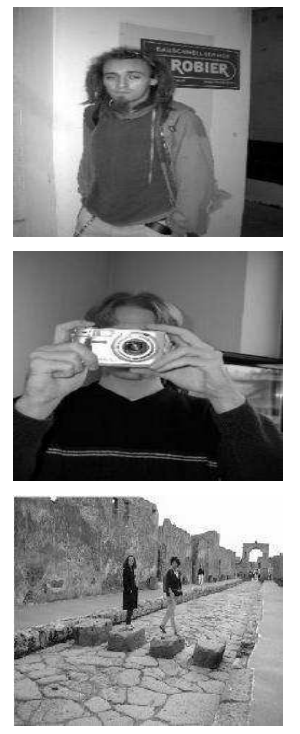

Human
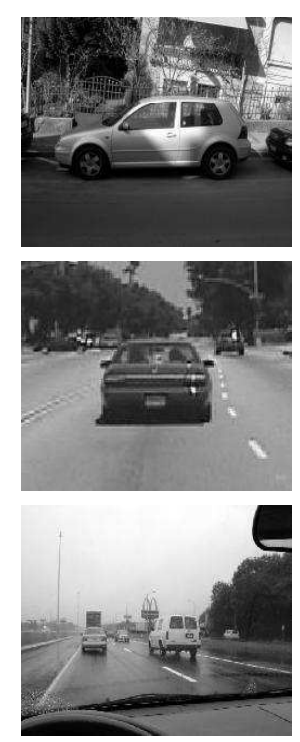

Car
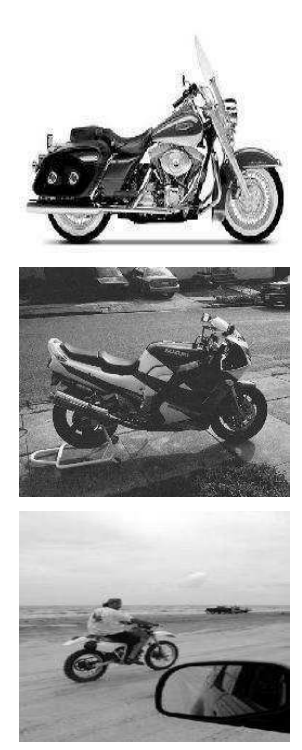

Motorbike

Figure 6: Some images used for learning and testing from the Pascal database [9].

come from a different distribution than the training set. An additional difficulty for test 2 is that many images contain instances of several categories. The Pascal dataset also provides for each image a bounding box indicating the localization of the object. The local image descriptors were obtained by first using the Harris-Laplace detector [22] to extract interest points and by then using the SIFT descriptor [20] to represent the scale-invariant regions around these points. The dimension of the obtained SIFT features is 128. See [6] for more details on the image descriptor extraction. Therefore, the object recognition task reduces to classifying the detected interest points in a 128-dimensional space. We evaluated our approach in both supervised and weakly supervised frameworks. In the supervised framework, only the descriptors located inside the bounding boxes were labeled as belonging to the class "object" in the learning step. Conversely, in the weakly supervised framework, all descriptors of images containing at least one instance of the object were labeled as belonging to the class "object" for learning. Figure 7 shows on the left panel an original image and on the right panel all extracted interest points. 

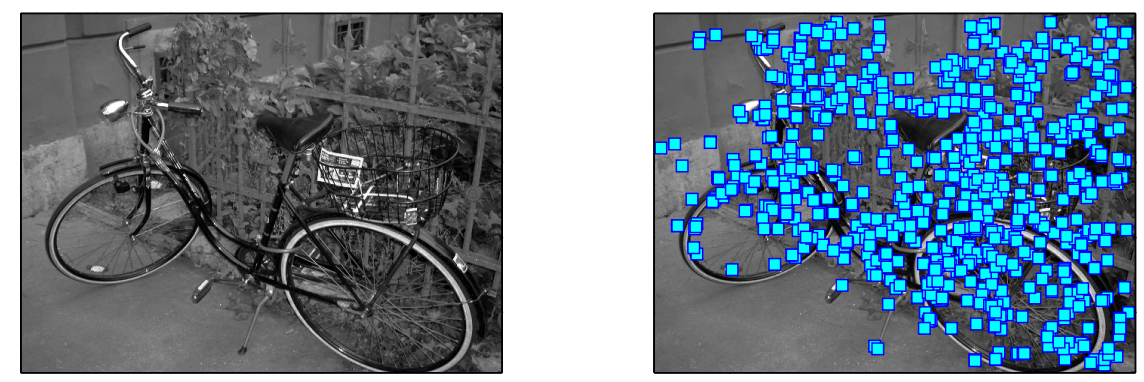

Figure 7: An original image from the Pascal test 1 dataset and the extracted interest points.

\subsection{Experimental setup}

We compared our approach based on RMDA with different Gaussian models to the object localization methods of the Pascal Challenge [9]. For RMDA, we used parsimonious Gaussian models as well as Gaussian models for highdimensional data, see Paragraph 2.2. On the one hand, we selected the following parsimonious Gaussian models: common covariance matrix Gaussian model (RMDA common), diagonal Gaussian model (RMDA diagonal), spherical Gaussian model (RMDA spherical). On the other hand, the following Gaussian models for high-dimensional data were also selected: $\left[a_{i j} b_{i} Q_{i} d_{i}\right]$, $\left[a_{i j} b Q_{i} d_{i}\right],\left[a_{i} b_{i} Q_{i} d_{i}\right],\left[a_{i} b Q_{i} d_{i}\right]$ and $\left[a_{i} b_{i} Q_{i} d\right]$. We refer to [5] for details on the different models. For all the models the parameters were estimated via the EM algorithm using the same initialization. For high-dimensional models, the resulting average value for intrinsic dimensions $d_{i}$ was approximately 10 . In this experiment, we used 50 clusters for modelling each of the four object categories whatever the clustering method used. In order to compare our results with the ones of the Pascal Challenge, we used the localization measure "Average Precision" (AP) proposed for this competition. It quantifies the consistency between the interest points classified as "object" with the provided bounding boxes (see [9] for more details). Therefore, the higher the $\mathrm{AP}$ value is, the better the object localization is.

\subsection{Experimental results}

Figure 8 illustrates the localization process with our approach in the weakly-supervised framework on an image from the test 1 dataset. Table 1 summarizes the localization results obtained in the supervised and weaklysupervised frameworks with our approach on both Pascal test 1 and test 2 

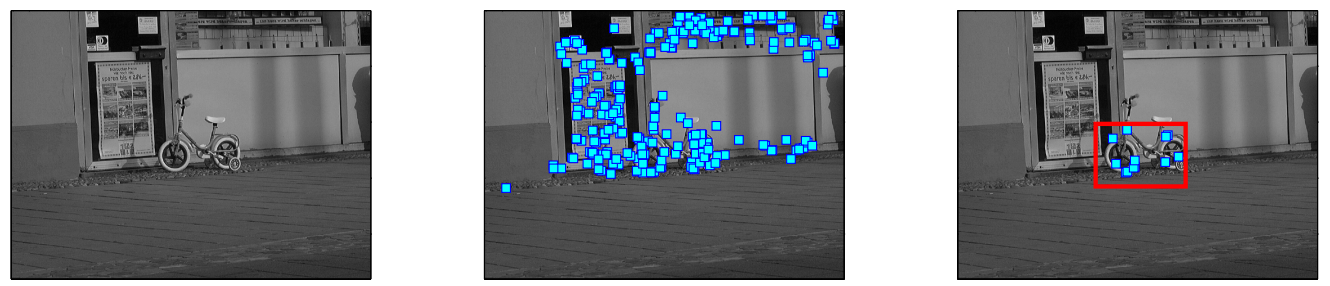

Figure 8: Weakly-supervised object localization on Pascal test 2: An original image from the Pascal test 1 dataset (left), the extracted interest points (center) and the interest points classified as "object" with the provided bounding box (right).

and reports the results of the best method of the Pascal challenge. The values presented in this table are the mean of the AP measure obtained by the different methods over the 4 object categories (motorbikes, bicycles, people and cars). Detailed results are presented in the appendix. On the one hand, our approach performs well in the supervised case compared to the results obtained during the Pascal competition. Moreover, the models designed for high-dimensional data perform best among the different Gaussian mixture models. In particular, RMDA with high-dimensional models wins two "competitions" (bicycle and people) on Pascal test 1 (see Appendix A.1) and three "competitions" (motorbike, bicycle and people) on Pascal test 2 (see Appendix A.2). This is despite the fact that our approach assumes that there is only one object per image for each category and this reduces the performance when multiple objects are present. On the other hand, it appears that the results obtained in the weakly-supervised framework are not very different from those obtained in the supervised framework. This means that our approach efficiently identifies discriminative clusters of each object category and that even with a weak supervision. We do not have the corresponding results for the Pascal challenge methods since there was no competition for detection in the weakly-supervised framework. However, we can remark that RMDA performs best in the weakly-supervised framework compared to the results of the Pascal Challenge methods in the supervised framework.

These results are actually promising and mean that the weakly-supervised approach is tenable for object localization since the manual annotation of training images is time consuming. Furthermore, this study has shown that classification with label noise can be extended to classification under weak supervision and that RMDA is able to solve both problems in complex situ- 


\begin{tabular}{|l|c|c|c|c|}
\hline Database & \multicolumn{2}{|c|}{ Pascal test 1 } & \multicolumn{2}{c|}{ Pascal test 2 } \\
\hline Supervision & full & weak & full & weak \\
\hline \hline RMDA $\left[a_{i j} b_{i} Q_{i} d_{i}\right]$ & 0.302 & 0.273 & 0.172 & 0.145 \\
RMDA $\left[a_{i j} b Q_{i} d_{i}\right]$ & $\mathbf{0 . 3 1 8}$ & $\mathbf{0 . 2 8 7}$ & 0.181 & 0.147 \\
RMDA $\left[a_{i} b_{i} Q_{i} d_{i}\right]$ & 0.313 & 0.285 & $\mathbf{0 . 1 8 3}$ & 0.142 \\
RMDA $\left[a_{i} b Q_{i} d_{i}\right]$ & $\mathbf{0 . 3 1 8}$ & 0.283 & 0.176 & $\mathbf{0 . 1 4 8}$ \\
RMDA $\left[a_{i} b_{i} Q_{i} d\right]$ & 0.314 & $\mathbf{0 . 2 8 7}$ & 0.179 & 0.130 \\
\hline RMDA spherical & 0.271 & 0.216 & 0.149 & 0.106 \\
RMDA diagonal & 0.276 & 0.227 & 0.161 & 0.110 \\
RMDA common & 0.267 & 0.246 & 0.164 & 0.116 \\
\hline Best method of $[9]$ & 0.279 & $/$ & 0.112 & $/$ \\
\hline
\end{tabular}

Table 1: Object localization results on the Pascal database: mean of the AP measure over the 4 object categories (motorbikes, bicycles, people and cars).

ations.

\section{Conclusion and discussion}

We have proposed in this paper a multi-class supervised classification method, called Robust Mixture Discriminant Analysis (RMDA), for performing classification in the presence of label noise. The experimental studies show that RMDA is as efficient as fully supervised techniques when the label noise is low and that RMDA is very robust to label noise, even in complex and real situations. In particular, RMDA appears to be more robust than existing methods. In addition, RMDA is able to deal with multi-class classification problems whereas existing methods cannot. Finally, we believe that this work may open the way to a new kind of learning in which a complete human supervision is not possible and replaced by a less expensive supervision. As an example, RMDA has been successfully applied for localizing objects in natural images in a weakly-supervised context which does not require the manual segmentation of the objects in many learning images. The classification method proposed in this paper could be therefore a way to solve an important issue of learning theory in the future: How to learn under weak supervision? 


\section{A. Appendix: detailed results for object localization}

\section{A.1. Object localization on Pascal test 1}

\begin{tabular}{|l|c|c|c|c|c|}
\hline Model & Motorbike & Bike & People & Car & Mean \\
\hline RMDA $\left[a_{i j} b_{i} Q_{i} d_{i}\right]$ & 0.665 & 0.403 & 0.047 & 0.095 & 0.302 \\
RMDA $\left[a_{i j} b Q_{i} d_{i}\right]$ & 0.680 & $\mathbf{0 . 4 3 9}$ & 0.032 & 0.123 & $\mathbf{0 . 3 1 8}$ \\
RMDA $\left[a_{i} b_{i} Q_{i} d_{i}\right]$ & 0.664 & 0.404 & 0.062 & 0.120 & 0.313 \\
RMDA $\left[a_{i} b Q_{i} d_{i}\right]$ & 0.671 & 0.437 & 0.035 & 0.128 & $\mathbf{0 . 3 1 8}$ \\
RMDA $\left[a_{i} b_{i} Q_{i} d\right]$ & 0.665 & 0.432 & $\mathbf{0 . 0 6 5}$ & 0.093 & 0.314 \\
\hline RMDA spherical & 0.572 & 0.349 & 0.042 & 0.118 & 0.271 \\
RMDA diagonal & 0.587 & 0.344 & 0.052 & 0.122 & 0.276 \\
RMDA common & 0.640 & 0.341 & 0.041 & 0.049 & 0.267 \\
\hline Best method of $[9]$ & $\mathbf{0 . 8 8 6}$ & 0.119 & 0.013 & $\mathbf{0 . 6 1 3}$ & 0.279 \\
\hline
\end{tabular}

Table 2: Object localization results (AP measure) in the supervised case on the Pascal test 1 database for the 4 object categories (motorbikes, bicycles, people and cars).

\begin{tabular}{|l|c|c|c|c|c|}
\hline Model & Motorbike & Bike & People & Car & Mean \\
\hline RMDA $\left[a_{i j} b_{i} Q_{i} d_{i}\right]$ & 0.658 & 0.384 & 0.020 & 0.027 & 0.273 \\
RMDA $\left[a_{i j} b Q_{i} d_{i}\right]$ & $\mathbf{0 . 6 8 6}$ & 0.404 & 0.038 & 0.020 & $\mathbf{0 . 2 8 7}$ \\
RMDA $\left[a_{i} b_{i} Q_{i} d_{i}\right]$ & 0.674 & 0.399 & 0.027 & $\mathbf{0 . 0 4 2}$ & 0.285 \\
RMDA $\left[a_{i} b Q_{i} d_{i}\right]$ & 0.671 & 0.403 & 0.022 & 0.034 & 0.283 \\
RMDA $\left[a_{i} b_{i} Q_{i} d\right]$ & 0.677 & $\mathbf{0 . 4 1 0}$ & 0.035 & 0.026 & $\mathbf{0 . 2 8 7}$ \\
\hline RMDA spherical & 0.592 & 0.228 & 0.021 & 0.025 & 0.216 \\
RMDA diagonal & 0.603 & 0.234 & $\mathbf{0 . 0 4 4}$ & 0.027 & 0.227 \\
RMDA common & 0.655 & 0.293 & 0.015 & 0.021 & 0.246 \\
\hline
\end{tabular}

Table 3: Object localization results (AP measure) in the weakly-supervised case on the Pascal test 1 database for the 4 object categories (motorbikes, bicycles, people and cars). 
A.2. Object localization on Pascal test 2

\begin{tabular}{|l|c|c|c|c|c|}
\hline Model & Motorbike & Bike & People & Car & Mean \\
\hline RMDA $\left[a_{i j} b_{i} Q_{i} d_{i}\right]$ & 0.305 & 0.169 & 0.061 & 0.154 & 0.172 \\
RMDA $\left[a_{i j} b Q_{i} d_{i}\right]$ & 0.316 & 0.164 & $\mathbf{0 . 0 9 1}$ & 0.151 & 0.181 \\
RMDA $\left[a_{i} b_{i} Q_{i} d_{i}\right]$ & 0.315 & $\mathbf{0 . 1 7 2}$ & $\mathbf{0 . 0 9 1}$ & 0.155 & $\mathbf{0 . 1 8 3}$ \\
RMDA $\left[a_{i} b Q_{i} d_{i}\right]$ & 0.307 & 0.169 & $\mathbf{0 . 0 9 1}$ & 0.136 & 0.176 \\
RMDA $\left[a_{i} b_{i} Q_{i} d\right]$ & 0.351 & 0.164 & 0.061 & 0.141 & 0.179 \\
\hline RMDA spherical & 0.261 & 0.142 & 0.045 & 0.149 & 0.149 \\
RMDA diagonal & 0.245 & 0.153 & 0.091 & 0.156 & 0.161 \\
RMDA common & 0.301 & 0.163 & 0.045 & 0.147 & 0.164 \\
\hline Best method of $[9]$ & $\mathbf{0 . 3 4 1}$ & 0.113 & 0.021 & $\mathbf{0 . 3 0 4}$ & 0.112 \\
\hline
\end{tabular}

Table 4: Object localization results (AP measure) in the supervised case on the Pascal test 2 database for the 4 object categories (motorbikes, bicycles, people and cars).

\begin{tabular}{|l|c|c|c|c|c|}
\hline Model & Motorbike & Bike & People & Car & Mean \\
\hline RMDA $\left[a_{i j} b_{i} Q_{i} d_{i}\right]$ & 0.304 & 0.141 & 0.021 & 0.115 & 0.145 \\
RMDA $\left[a_{i j} b Q_{i} d_{i}\right]$ & 0.312 & 0.141 & 0.018 & 0.115 & 0.147 \\
RMDA $\left[a_{i} b_{i} Q_{i} d_{i}\right]$ & 0.311 & $\mathbf{0 . 1 6 1}$ & $\mathbf{0 . 0 4 5}$ & 0.049 & 0.142 \\
RMDA $\left[a_{i} b Q_{i} d_{i}\right]$ & 0.298 & 0.153 & 0.026 & $\mathbf{0 . 1 1 6}$ & $\mathbf{0 . 1 4 8}$ \\
RMDA $\left[a_{i} b_{i} Q_{i} d\right]$ & $\mathbf{0 . 3 2 2}$ & 0.141 & 0.023 & 0.034 & 0.130 \\
\hline RMDA spherical & 0.254 & 0.111 & 0.023 & 0.037 & 0.106 \\
RMDA diagonal & 0.239 & 0.120 & 0.011 & 0.069 & 0.110 \\
RMDA common & 0.276 & 0.142 & 0.008 & 0.036 & 0.116 \\
\hline
\end{tabular}

Table 5: Object localization results (AP measure) in the weakly-supervised case on the Pascal test 2 database for the 4 object categories (motorbikes, bicycles, people and cars). 


\section{References}

[1] J. Banfield and A. Raftery. Model-based Gaussian and non-Gaussian clustering. Biometrics, 49:803-821, 1993.

[2] S. Bashir and E. Carter. High breakdown mixture discriminant analysis. Journal of Multivariate Analysis, 93(1):102-111, 2005.

[3] R. Bellman. Dynamic programming. Princeton University Press, 1957.

[4] H. Bensmail and G. Celeux. Regularized Gaussian discriminant analysis through eigenvalue decomposition. Journal of the American Statistical Association, 91:1743-1748, 1996.

[5] C. Bouveyron, S. Girard, and C. Schmid. High-Dimensional Data Clustering. Computational Statistics and Data Analysis, 52(1):502-519, 2007.

[6] C. Bouveyron, J. Kannala, C. Schmid, and S. Girard. Object localization by subspace clustering of local descriptors. In 5th Indian Conference on Computer Vision, Graphics and Image Processing, pages 457-467, India, 2006.

[7] C. Brodley and M. Friedl. Identifying mislabeled training data. Journal of Artificial Intelligence Research, 11:131-167, 1999.

[8] G. Celeux and G. Govaert. Parsimonious Gaussian models in cluster analysis. Pattern Recognition, 28:781-793, 1995.

[9] F. d'Alche Buc, I. Dagan, and J. Quinonero, editors. The 2005 Pascal visual object classes challenge. Proceedings of the first PASCAL Challenges Workshop. Springer, 2006.

[10] B. Dasarathy. Noising around the neighbourhood: a new system structure and classification rule for recognition in partially exposed environments. IEEE Trans. Pattern Analysis and Machine Intelligence, 2:67-71, 1980.

[11] D. Gamberger, N. Lavrac, and C. Groselj. Experiments with noise filtering in a medical domain. In 16th International Conference on Machine Learning, pages 143-151, USA, 1999. 
[12] G. Gates. The reduced nearest neighbor rule. IEEE Transactions on Information Theory, 18(3):431-433, 1972.

[13] I. Guyon, N. Matic, and V. Vapnik. Discovering informative patterns and data cleaning. Advances in Knowledge Discovery and Data Mining, pages 181-203, 1996.

[14] T. Hastie and R. Tibshirani. Discriminant analysis by Gaussian mixtures. Journal of the Royal Statistical Society B, 58:155-176, 1996.

[15] T. Hastie, R. Tibshirani, and J. Friedman. The elements of statistical learning. Springer, New York, 2001.

[16] D. Hawkins and G. McLachlan. High-breakdown linear discriminant analysis. Journal of the American Statistical Association, 92(437):136$143,1997$.

[17] G. John. Robust decision trees: Removing outliers from databases. In First conference on Knowledge Discovery and Data Mining, pages 174179, 1995.

[18] N. Lawrence and B. Schölkopf. Estimating a kernel Fisher discriminant in the presence of label noise. In Proc. of 18th International Conference on Machine Learning, pages 306-313. Morgan Kaufmann, San Francisco, CA, 2001.

[19] Y. Li, L. Wessels, D. de Ridder, and M. Reinders. Classification in the presence of class noise using a probabilistic kernel Fisher method. Pattern Recognition, 40(12):3349-3357, 2007.

[20] D. Lowe. Distinctive image features from scale-invariant keypoints. International Journal of Computer Vision, 60(2):91-110, 2004.

[21] G. McLachlan. Discriminant Analysis and Statistical Pattern Recognition. Wiley, New York, 1992.

[22] K. Mikolajczyk and C. Schmid. Scale and affine invariant interest point detectors. International Journal of Computer Vision, 60(1):63-86, 2004.

[23] J. Mingers. An empirical comparison of pruning methods for decision tree induction. Journal of Machine Learning, 4(2):227-243, 1989. 
[24] J. Quinlan. Bagging, boosting and C4.5. In 13th National Conference on Artificial Intelligence, pages 725-730, USA, 1996.

[25] P.J. Rousseeuw and A. Leroy. Robust Regression and Outlier Detection. Wiley, New York, 1987.

[26] Y. Sakakibara. Noise-tolerant occam algorithms and their applications to learning decision trees. Journal of Machine Learning, 11(1):37-62, 1993.

[27] R. Schapire. The strength of weak learnability. Machine Learning, 5:197227, 1990.

[28] G. Schwarz. Estimating the dimension of a model. The Annals of Statistics, 6:461-464, 1978.

[29] P. Vannoorenbergue and T. Denoeux. Handling uncertain labels in multiclass problems using belief decision trees. In Proceedings of IPMU'2002, 2002.

[30] D. Wilson and T. Martinez. Instance pruning techniques. In Fourteenth International Conference on Machine Learning, pages 404-411, USA, 1997.

[31] X. Zeng and T. Martinez. A noise filtering method using neural networks. In IEEE International Workshop on Soft Computing Techniques in Instrumentation, Measurement and Related Applications, pages 2631, 2003.

[32] X. Zhu, X. Wu, and Q. Chen. Eliminating class noise in large datasets. In 20th ICML International Conference on Machine Learning, pages 920-927, USA, 2003. 